\title{
Tubularised Incised Plate Urethroplasty is an Applicable Method for the Treatment of the Hypospadiac Type of Urethral Duplication
}

\author{
Ömer Yılmaz, Cüneyt Günşar
}

Department of Pediatric Surgery, Facult of Medicine, Celal Bayar University, Manisa, Turkey

\begin{abstract}
Urethral duplication is a rare congenital anomaly usually seen in males. We present a case of urethral duplication treated by a tubularised incised plate urethroplasty (TIPU) technique. A 5-year-old male boy was admitted to the clinic with the complaints of thin calibrated urinating and diurnal enuresis. We diagnosed distal penile hypospadias. During the operation, another urethral opening was found proximal to the previously seen hypospadiac meatus, localised on the coronary sulcus. We performed the TIPU procedure without excising the duplicated urethra. We only excised the common wall between the two urethras, and used the dorsal urethral plate of the duplicated urethra for tubularisation of the neourethra. The postoperative course was uneventful. A cystoscopy was done for suspected urogenital sinus. There was no further complaint at the third postoperative month. The TIPU technique can be successfully applied in such cases. The dorsal plate of the duplicated urethra can be used to form the neourethra.
\end{abstract}

Key Words: Urethral duplication, hypospadias, TIPU

\section{Introduction}

Urethral duplication is a rare congenital anomaly that is usually seen in males and, in rare cases, in females. Several different anatomical variations have been described. Sagittal localisation is the most common type of urethral duplication that usually develops as an isolated malformation. Coronal duplications, on the other hand, are rarer and are found in association with bladder duplications (1). Almost all of the female patients with urethral duplications also have double bladders (2). The duplicated urethra is often incomplete and remains dorsal to the true urethra in the sagittal plane. The ventral meatus (generally supplying the urinary outflow as a true urethral opening) may be located at any point on the ventral side of the penis. We present a case of hypospadiac type incomplete duplicated urethra treated by a tubularised incised plate urethroplasty technique.

\section{Case}

A 5-year-old male boy was admitted to the clinic with the complaints of thin calibrated urinating and diurnal enuresis. We diagnosed distal penile hypospadias. The urethral meatus was localised in the midline on the coronary sulcus. The glandular groove was prominent. Blood tests and urinary analysis were normal. We decided to perform a tubularised incised plate urethroplasty (TIPU). The penile skin was freed by incising circumferentially from just below the meatus, which was localised on the coronary sulcus. During the dissection an- other urethral opening was seen at the ventral localisation (Fig. 1). This meatus was catheterised up to the bladder and urinary discharge was observed. The incomplete dorsal urethra had a $3 \mathrm{~cm}$ blind lumen (Fig. 2). An erection test was done after penile degloving by injecting physiological saline, and no chordee was present. Then the ventral wall of the dorsal urethra was excised longitudinally and the common wall between the two urethras was incised. Two parallel vertical incisions were made bilaterally to the urethral plate of the dorsal abortive urethra extending to the glans. The glandular wings were freed by sharp dissection. A midline vertical incision of the urethral plate was made beginning from the tip of the glans down to the urethral meatus. The bladder was catheterized with six French feeding tubes. The dissected urethral plate was anteriorly tubularised with 6/0 PDS running subcuticular sutures. The suture line was strengthened with another row of sutures of the same material. Then a pedunculated flap was prepared from the inner tissues of the preputium. It was easily mobilised. A button-hole perforation was made on the proximal part of the flap and it was turned down to the ventral side of the penis. A flap covered the tubularised urethra and was sutured bilaterally to the inner tissues of the glans. Then the glans wings were closed with a $5 / 0$ vicryl suture, first subepithelial, and then with a second row of skin suturing. The operation was completed with circumferential suturing of the penile skin.

The postoperative course was uneventful. After 7 days, the catheter was removed and the child urinated spontaneously. Two weeks after surgery, an urethrogram was per- 


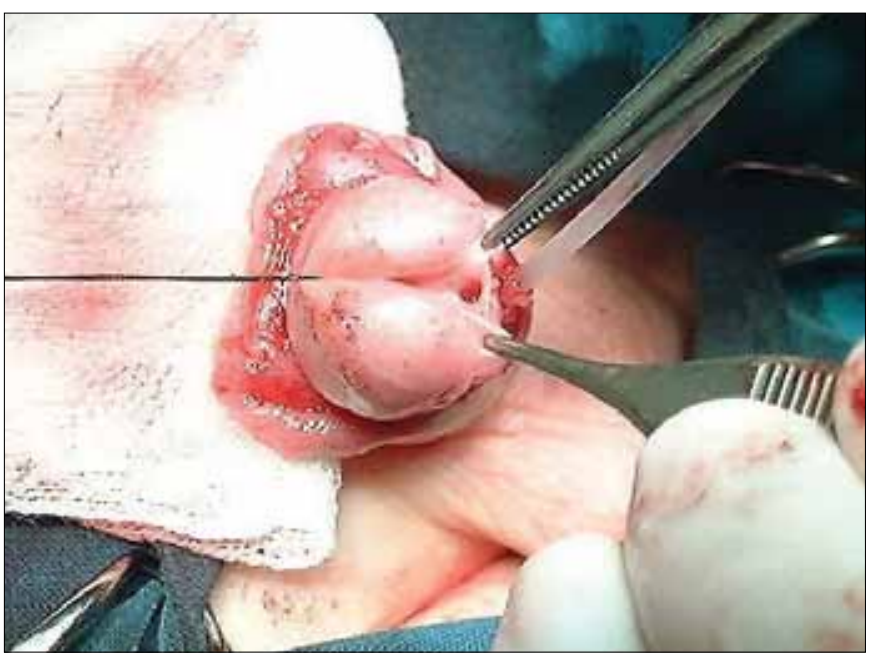

Figure 1. Intraoperative view of the hypospadiac ventral urethral orifice and duplicated urethral meatus

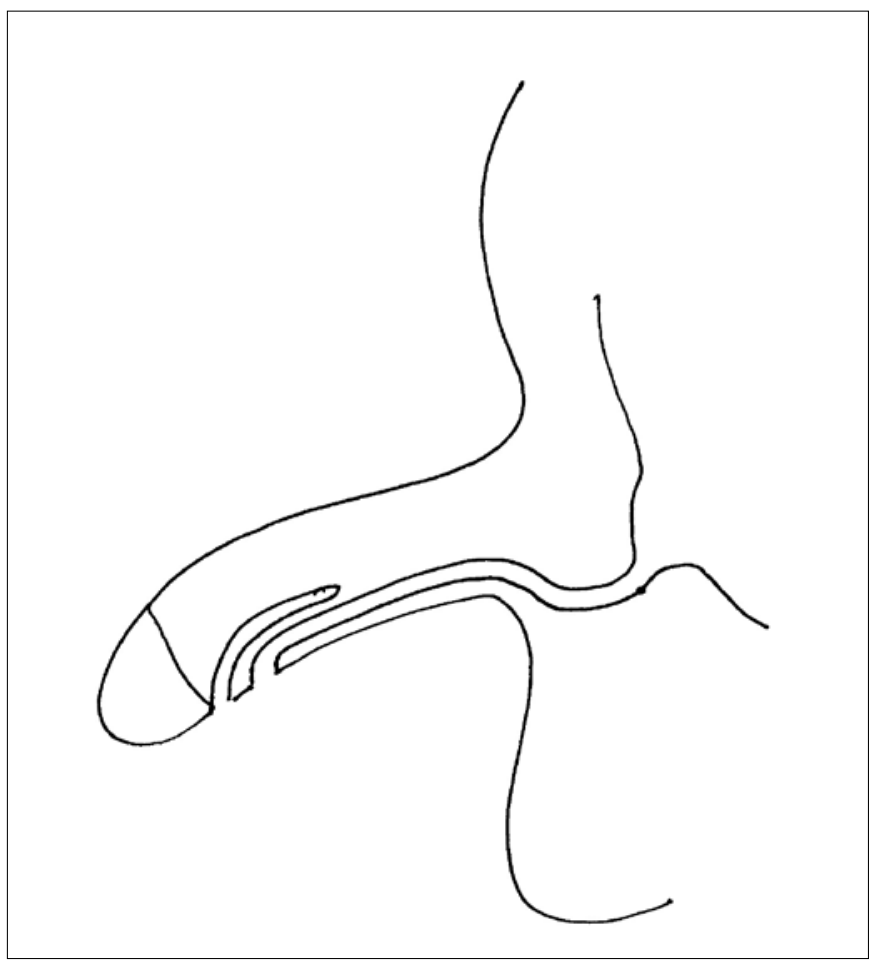

Figure 2. The depicted figure shows the incompletely duplicated urethra dorsally, and the complete hypospadiac urethra at the ventral position in the sagittal view

formed and overfilling was seen in the prosthetic urethra with a length of $1 \mathrm{~cm}$ and a width of $3 \mathrm{~mm}$. Urinary system ultrasonography revealed no pathology other than a minimally thickened bladder wall. The patient was examined 3 weeks after surgery. The urine calibration was normal. There was no daytime incontinence; the nighttime incontinence was evaluated as partially healed. Urethral meatal dilatation (up to the 12 French and cystoscopy (the proximal urethra was dilated and the verumontanum was prominent) were performed under general anaesthesia. There were no further complaints 3 months after the operation.

\section{Discussion}

Urethral duplication is a rare congenital anomaly and hypospadiac types are sometimes found incidentally and their anatomical characteristics identified during surgery (1). In 1975, Effman et al. (3) described a detailed classification of urethral duplications depending on localisation, the dominant urethra and meatal position. Our patient had a Type 1 anomaly according to this classification. Although the mechanism of development of the anomaly is not clearly understood, several theories have been suggested, such as: abnormal termination of the Mullerian duct (4), asymmetry in the closure of the urorectal septum (5), and partial failure or an irregularity in the ingrowth of the lateral mesoderm between the ectodermal and endodermal layers of the cloacal membrane in the midline (6). However, none of the theories alone are sufficient to explain the whole anomaly spectrum.

Urethral duplications may be epispadiac, hypospadiac or "Y" type. In addition, complete or incomplete forms may be seen. Surgery is indicated in hypospadiac types. Operational techniques include repair of the hypospadiac urethra and the excision of the duplicated urethra (1). It has been reported that the duplicated urethra should be excised because of its hypoplastic nature. However, in 2005, Etensel et al. (7) published their case of urethral duplication with a hypospadias anomaly, which was very similar to our case. This was the first case of urethral duplication treated with the Snodgrass procedure and reported in the English literature. They thoroughly excised the common channel and repaired the defect using the TIPU technique. As far as we know, our patient is the second case to be treated by the same method. This procedure allows use of the dorsal plate of the distal urethra, which is usually abortive. This technique is not a modification. After excising the ventral side of the distal urethra and excising the common wall to combine the two urethral lumens, the steps used in the original TIPU procedure are applied for the repair.

Three months after the operation the patient had no complaints of enuresis. This may be coincidental, or may be related to the healing of the stenotic hypospadiac urethral orifice.

Snodgrass initially developed his operation (TIPU) for the treatment of the distal hypospadias forms (8), but later, he and others successfully used this technique for the treatment of proximally located types of hypospadias (9). Its cosmetic properties in terms of the meatal appearance and localisation are satisfactory. We showed that this technique can be used for the hypospadiac type of urethral duplication. In our patient, the orifices were more proximally located when compared with the case of Etensel et al. (7). The abortive urethral meatus was described as localising more distally, on the glandular groove in their case, whereas it was located on the coronary sulcus in our patient. This technique has all the advantages of the classical 'Snodgrass' technique, and there is no need for excision of the duplicated urethra, which may be difficult.

Urethral duplication is generally associated with duplication of other genital organs, the penis, bladder, etc. and is associated with other urinary system anomalies. We therefore scanned the urinary system with voiding cystourethrography and ultrasonography and found no associated anomaly. 
In conclusion, the hypospadiac type of abortive urethral duplication is a very rare anomaly that is generally found incidentally during surgery. The ideal surgical treatment of urethral duplication anomalies remains uncertain. Although the postoperative follow-up of the case was not long enough to observe any complications of the operation, the TIPU operation is a suitable method for the treatment of this type of anomaly combined with the incision of the common wall between the two urethras and the use of the dorsal plate of the duplicated urethra to form the neourethra.

\section{Conflict of Interest}

No conflict of interest was declared by the authors.

\section{References}

1. Salle JL, Sibai H, Rosenstein D, Brzezinski AE, Corcos J. Urethral duplication in the male: Review of 16 cases. J Urol 2000;163:1936-40. [CrossRef]
2. Prasad N,Vivekanandhan KG, llangovan G, Prabakaran S. Duplication of the urethra. Pediatr Surg Int 1999;15:419-21. [CrossRef]

3. Effman EL, Lebowitz RL, Colodny AH. Duplication of the urethra. Radiology 1976;119:179-85.

4. Das S, Brosman SA. Duplication of the male urethra. J Urol 1997;117:452-4.

5. Rica PE, Holder TM, Ashcraft KW. Congenital posterior urethral perineal fistula: A case report. J Urol 1978;199:416-7.

6. Casselman J, Williams DI. Duplication of the urethra. Acta Urol Belg 1996;34:535-41.

7. Etensel B, Yazıcı M, Gursoy H, Ozkisacik S. Tubularised incised plate urethroplasty for abortive hypospadiac urethral duplication. Pediatr Surg Int 2005;21:494-6. [CrossRef]

8. Snodgrass WT. Tubularized incised-plate hypospadias repair: indications, technique, and complications. Urology 1999;54:6-11. [CrossRef]

9. Snodgrass WT, Lorenzo A. Tubularised incised-plate urethroplasty for proximal hypospadias. BJU Int 2002;89:90-3. [CrossRef] 